\title{
Erratum zu: Wesentliche Arbeitsmerkmale
}

\section{Erratum zu: Kapitel 4 in: V. Mustapha und F. Schweden, Arbeitsanalyse - Arbeitsbewertung - Arbeitsgestaltung,essentials, https://doi.org/10.1007/978-3-658-33129-0_4}

Dieses Buch wurde versehentlich veröffentlicht ohne nachstehenden Text in Kapitel 4.2 korrigierend zu ergänzen:

Beispiel 4.1 zur Unterscheidung von task identity des Job Characteristics Model und der vollständigen Tätigkeit der Handlungsregulationstheorie (aus Mustapha 2020)

Das JCM weist fünf Kernmerkmalen auf, welche in Kombination zu hoher intrinsischer Motivation und Arbeitsleistung führen sollen. Ein zentrales Merkmal ist dabei die task identity (Definition: „the degree to which the job requires completion of a 'whole' and identifiable piece of work; that is, doing a job from beginning to end with a visible outcome", Hackman und Oldham 1976, S. 275). Demnach ist task identity ein eng gefasstes und durch die andere Kernmerkmale beeinflussbares Konstrukt. Hingegen ist die Bedeutung des Konstrukts der vollständigen Tätigkeit auf Basis der Handlungsregulationstheorie wesentlich umfassender und geht über das ,doing a job from beginning to end" (Hackman und Oldham 1976, S. 275) hinaus. Deutlich werden die

Die korrigierte Version des Kapitels ist verfügbar https://doi.org/10.1007/978-3-658-33129-0_4 
Unterschiede beider theoretischer Konstrukte an einem Beispiel: Stellen Sie sich vor, dass Ihr Auftrag darin besteht, einen Schaukelstuhl mit den vorhandenen Bauteilen und Werkzeugen nach vorgegebenen Regeln zu fertigen. Ihr Arbeitsplatz hält dabei alle Bauteile, Werkzeuge sowie detaillierte Fertigungsvorschriften für sie bereit. Folglich können Sie den Schaukelstuhl problemlos zusammenbauen. Nach dem JCM liegt bei diesem Beispiel ein ,whole piece of work" (Hackman und Oldham 1976, S. 275) und somit task identity vor. Im Sinne der Handlungsregulationstheorie hält dieses Beispiel allerdings nur die Tätigkeitsklasse Ausführen für Sie bereit. In der Theorie wäre diese Tätigkeit somit sequentiell und hierarchisch unvollständig. Wenn Sie jedoch den Auftrag erhalten würden, dass Sie einen Schaukelstuhl konstruieren, fertigen und anschließend auf Funktionalität überprüfen sollen, wäre die Tätigkeit sequentiell vollständig. Sequentielle Vollständigkeit liegt vor, da Sie Ihre eigene Tätigkeit planen, vorbereiten sowie organisatorische Abstimmungen mit anderen Arbeitenden treffen müssen und schließlich das Arbeitsergebnis (den Schaukelstuhl) eigenständig oder mithilfe von Rückmeldungen kontrollieren können. Durch die Möglichkeit zum Entwurf des Schaukelstuhls, wäre auch schöpferisches Denken bzw. die intellektuelle Regulationsebene gegeben. Die Tätigkeit wäre somit auch hierarchisch vollständig. Eine sequentiell und hierarchisch vollständige Tätigkeit bedingt wiederum Arbeitsmerkmale wie Verantwortungsübernahme, Kooperations- und Lernmöglichkeiten. Die Tätigkeit erfüllt folglich alle Merkmale einer gut gestalteten Arbeit nach DIN EN ISO 6385 (2016). 